\title{
SDHB/SDHA immunohistochemistry in pheochromocytomas and paragangliomas: a multicenter interobserver variation analysis using virtual microscopy: a Multinational Study of the European Network for the Study of Adrenal Tumors (ENS@T)
}

Thomas G Papathomas ${ }^{1,2}$, Lindsey Oudijk ${ }^{2}$, Alexandre Persu ${ }^{3}$, Anthony J Gill ${ }^{4,5}$, Francien van Nederveen ${ }^{6}$, Arthur S Tischler ${ }^{7}$, Frédérique Tissier ${ }^{8,9}$, Marco Volante ${ }^{10}$, Xavier Matias-Guiu ${ }^{11}$, Marcel Smid ${ }^{12}$, Judith Favier ${ }^{13,14}$, Elena Rapizzi ${ }^{15}$, Rosella Libe ${ }^{8}$, Maria Currás-Freixes ${ }^{16}$, Selda Aydin ${ }^{17}$, Thanh Huynh ${ }^{18}$, Urs Lichtenauer ${ }^{19}$, Anouk van Berkel ${ }^{20}$, Letizia Canu ${ }^{15}$, Rita Domingues ${ }^{21}$, Roderick J Clifton-Bligh ${ }^{22}$, Magdalena Bialas ${ }^{23}$, Miikka Vikkula ${ }^{24}$, Gustavo Baretton ${ }^{25}$, Mauro Papotti ${ }^{10}$, Gabriella Nesi ${ }^{26}$, Cécile Badoual ${ }^{13,14,27}$, Karel Pacak ${ }^{18}$, Graeme Eisenhofer ${ }^{28}$, Henri J Timmers ${ }^{20}$, Felix Beuschlein ${ }^{19}$, Jérôme Bertherat ${ }^{29,30}$, Massimo Mannelli ${ }^{15,31}$, Mercedes Robledo ${ }^{16,32}$, Anne-Paule Gimenez-Roqueplo ${ }^{13,14}$, Winand NM Dinjens ${ }^{2}$, Esther Korpershoek ${ }^{2}$ and Ronald R de Krijger ${ }^{2,33,34}$

${ }^{1}$ Department of Histopathology, King's College Hospital, London, UK; ${ }^{2}$ Department of Pathology, Erasmus MC Cancer Institute, University Medical Center Rotterdam, Rotterdam, The Netherlands; ${ }^{3}$ Pole of Cardiovascular Research, Institut de Recherche Expérimentale et Clinique and Division of Cardiology, Cliniques Universitaires Saint-Luc, Université Catholique de Louvain, Brussels, Belgium; ${ }^{4}$ Department of Anatomical Pathology, Royal North Shore Hospital, St Leonards, NSW, Australia; ${ }^{5}$ Cancer Diagnosis and Pathology Research Group, Kolling Institute of Medical Research, University of Sydney, Sydney, NSW, Australia; ${ }^{6}$ Laboratory for Pathology, PAL Dordrecht, Dordrecht, The Netherlands; ${ }^{7}$ Department of Pathology and Laboratory Medicine, Tufts Medical Center, Tufts University School of Medicine, Boston, MA, USA; ${ }^{8}$ Institut National de la Santé et de la Recherche Médicale U1016, Institut Cochin, Centre National de la Recherche Scientifique UMR8104, Université Paris Descartes, Sorbonne Paris Cité, Rare Adrenal Cancer Network COMETE, Paris, France; ${ }^{9}$ Department of Pathology, Hôpital Pitié-Salpêtrière, Université Pierre et Marie Curie, Paris, France; ${ }^{10}$ Department of Oncology, University of Turin at San Luigi Hospital, Turin, Italy; ${ }^{11}$ Department of Pathology and Molecular Genetics and Research Laboratory, Hospital Universitari Arnau de Vilanova, IRBLLEIDA, University of Lleida, Lleida, Spain; ${ }^{12}$ Department of Medical Oncology, Erasmus MC Cancer Institute, University Medical Center Rotterdam, Rotterdam, The Netherlands; ${ }^{13}$ Paris-Centre de Recherche Cardiovasculaire (PARCC), Inserm UMR970, Hôpital Européen Georges Pompidou, Paris, France; ${ }^{14}$ Université Paris Descartes, Faculté de Médecine, Paris Cité Sorbonne, France; ${ }^{15}$ Endocrinology Unit, Department of Experimental and Clinical Biomedical Sciences, University of Florence, Florence, Italy; ${ }^{16}$ Hereditary Endocrine Cancer Group, Spanish National Cancer Research Centre (CNIO), Madrid, Spain; ${ }^{17}$ Department of Pathology, Cliniques Universitaires Saint-Luc, Université catholique de Louvain, Institut de Recherche Expérimentale et Clinique, Brussels, Belgium; ${ }^{18}$ Program in Reproductive and Adult Endocrinology, Eunice Kennedy Shriver National Institute of Child Health and Human Development, National Institutes of Health, Bethesda, MD, USA; ${ }^{19}$ Endocrine Research Unit, Medizinische Klinik und Poliklinik IV, Klinikum der Universität München, Munich, Germany; ${ }^{20}$ Department of Internal Medicine, Section of Endocrinology, Radboud University Medical Centre, Nijmegen, The Netherlands; ${ }^{21}$ Unidade de Investigação em Patobiologia Molecular (UIPM), Instituto Português de Oncologia de Lisboa Francisco Gentil, Lisbon, Portugal; ${ }^{22}$ Cancer Genetics, Kolling Institute of Medical Research, Royal North Shore Hospital, University of Sydney, Sydney, NSW, Australia; ${ }^{23}$ Department of Pathomorphology, Jagiellonian University Medical College, 
Krakow, Poland; ${ }^{24}$ Laboratory of Human Molecular Genetics, de Duve Institute, Université Catholique de Louvain, Brussels, Belgium; ${ }^{25}$ Department of Pathology, Technische Universität Dresden, Dresden, Germany; ${ }^{26}$ Division of Pathological Anatomy, University of Florence, Florence, Italy; ${ }^{27}$ Service d'Anatomie Pathologique, Hôpital Européen Georges-Pompidou, Assistance Publique Hôpitaux de Paris (AP-HP), Paris, France; ${ }^{28}$ Institute of Clinical Chemistry and Laboratory Medicine and Department of Medicine III, University Hospital, Technische Universität Dresden, Dresden, Germany; ${ }^{29}$ Institut Cochin, Université Paris Descartes, INSERM U1016, CNRS UMR8104, Paris, France; ${ }^{30}$ Department of Endocrinology, Referral Center for Rare Adrenal Diseases, Assistance Publique Hôpitaux de Paris, Hôpital Cochin, Paris, France; ${ }^{31}$ Istituto Toscano Tumori (ITT), Florence, Italy; ${ }^{32}$ Centre for Biomedical Network Research on Rare Diseases (CIBERER), Madrid, Spain; ${ }^{33}$ Department of Pathology, Reinier de Graaf Hospital, Delft, The Netherlands and ${ }^{34}$ Department of Pathology, University Medical Center Utrecht, Princess Maxima Center for Pediatric Oncology, Utrecht, The Netherlands

Despite the established role of SDHB/SDHA immunohistochemistry as a valuable tool to identify patients at risk for familial succinate dehydrogenase-related pheochromocytoma/paraganglioma syndromes, the reproducibility of the assessment methods has not as yet been determined. The aim of this study was to investigate interobserver variability among seven expert endocrine pathologists using a web-based virtual microscopy approach in a large multicenter pheochromocytoma/paraganglioma cohort $(n=351)$ : (1) 73 SDH mutated, (2) 105 non-SDH mutated, (3) 128 samples without identified SDH-x mutations, and (4) 45 with incomplete SDH molecular genetic analysis. Substantial agreement among all the reviewers was observed either with a two-tiered classification (SDHB $\kappa=0.7338$; SDHA $\kappa=0.6707$ ) or a three-tiered classification approach (SDHB $\kappa=0.6543$; SDHA $K=0.7516)$. Consensus was achieved in 315 cases $(89.74 \%)$ for SDHB immunohistochemistry and in 348 cases $(99.15 \%)$ for SDHA immunohistochemistry. Among the concordant cases, 62 of $69(\sim 90 \%)$ SDHB-/C-ID-I AF2-mutated cases displayed SDHB immunonegativity and SDHA immunopositivity, 3 of $4(75 \%)$ with SDHA mutations showed loss of SDHA/SDHB protein expression, whereas 98 of 105 (93\%) non-SDH-x-mutated counterparts demonstrated retention of SDHA/SDHB protein expression. Two SDHD-mutated extra-adrenal paragangliomas were scored as SDHB immunopositive, whereas 9 of $128(7 \%)$ tumors without identified SDH-x mutations, 6 of $37(\sim 16 \%)$ VHL-mutated, as well as 1 of $21(\sim 5 \%)$ NF1-mutated tumors were evaluated as SDHB immunonegative. Although 14 out of those 16 SDHB-immunonegative cases were nonmetastatic, an overall significant correlation between SDHB immunonegativity and malignancy was observed $(P=0.00019)$. We conclude that SDHB/SDHA immunohistochemistry is a reliable tool to identify patients with $S D H-x$ mutations with an additional value in the assessment of genetic variants of unknown significance. If SDH molecular genetic analysis fails to detect a mutation in SDHB-immunonegative tumor, SDHC promoter methylation and/or VHLNF1 testing with the use of targeted next-generation sequencing is advisable.

Modern Pathology (2015) 28, 807-821; doi:10.1038/modpathol.2015.41; published online 27 February 2015

Pheochromocytomas and paragangliomas are neural crest-derived neuroendocrine tumors arising from the adrenal medulla and sympathetic/parasympathetic paraganglia, respectively. ${ }^{1}$ These carry the highest degree of heritability among human neoplasms. Germline and/or somatic mutations of at least 18 genes (NF1, RET, VHL, SDHA, SDHB, SDHC, SDHD, SDHAF2, TMEM127, MAX, HIF2A, KIF1B, PHD1, PHD2/EGLN1, FH, HRAS, BAP1, and MEN1) are involved in development of the tumors, with $\sim 40 \%$ harboring a germline mutation and an additional $25-30 \%$ a somatic mutation. ${ }^{2-4}$

Correspondence: Dr TG Papathomas, MD, Department of Histopathology, King's College Hospital, Denmark Hill, London, UK. E-mail: t.papathomas@erasmusmc.nl or thomaspapathomas@nhs. net

Received 26 October 2014; revised 10 January 2015; accepted 10 January 2015; published online 27 February 2015
Familial succinate dehydrogenase-related pheochromocytoma/paraganglioma syndromes are caused by $S D H A, S D H B, S D H C, S D H D$, and SDHAF2 (collectively $S D H-x$ ) mutations and inherited as autosomal dominant traits. ${ }^{4}$ These syndromes predispose not only to pheochromocytomas/paragangliomas, but also to gastrointestinal stromal tumors, renal cell carcinomas, and pituitary adenomas. ${ }^{5-7}$ In the vast majority of succinate dehydrogenaseassociated tumors, there is also loss of SDHB and/ or SDHA protein expression that can be detected by immunohistochemistry. ${ }^{5-41}$ In particular, $S D H B-$, $S D H C$-, and $S D H D$-mutated tumors display SDHB immunonegativity but SDHA immunoreactivity, whereas SDHA-mutated tumors show negativity for both SDHB and SDHA immunostainings. Gastrointestinal stromal tumors and paragangliomas, associated with Carney triad (the syndromic but nonhereditary association of gastrointestinal stromal 
tumor, paraganglioma, pulmonary chondroma, adrenocortical adenoma, and esophageal leiomyoma), ${ }^{4}$ show negative staining for SDHB in the absence of $S D H-x$ mutations. ${ }^{29,40}$ There is provisional evidence that Carney triad-related tumors display somatic hypermethylation of the $S D H C$ promoter locus, ${ }^{42}$ and therefore negative staining for SDHB may also identify these cases not found by conventional molecular testing.

As loss of SDHB/ SDHA expression is predictive of an underlying $S D H-x$ germline mutation, ${ }^{8,10,11,17,21-24,29,34,39}$ the role of SDHB/ SDHA immunohistochemistry has been underlined as a supplementary approach in molecular genetic testing especially for pheochromocytomas and paragangliomas. ${ }^{8,10,11}$ As Sanger or targeted nextgeneration sequencing analysis of all pheochromocytoma/paraganglioma susceptibility genes is labor intensive and/or requires clinical molecular diagnostic laboratories, ${ }^{43-45}$ it might be prudent to use immunohistochemistry to identify patients with succinate dehydrogenase-related pheochromocytoma/paraganglioma syndromes. In addition, the presence of an $S D H B$ mutation is one of the strongest predictors for both metastasis and subsequently poor outcome in pheochromocytomas/paragangliomas. ${ }^{4}$ In this context, it has been proposed that a combination of the GAPP (grading system for adrenal phaeochromocytoma and paraganglioma) and SDHB immunohistochemistry might be a valuable aid in the prediction of metastatic disease, ${ }^{46}$ further necessitating correct interpretation of SDHB/SDHA immunostainings.

Given the high prevalence of unsuspected hereditary disease, false-positive as well as false-negative evaluations of SDHB/SDHA immunostainings can lead to failure to identify pheochromocytoma/paraganglioma-affected individuals at increased risk for succinate dehydrogenase-related neoplasia, incorrect interpretation of the pathogenicity of genetic variants of uncertain significance, and inappropriate genetic testing. Because studies addressing the issue of interobserver variation for SDHB/SDHA immunohistochemistry in pheochromocytomas/paragangliomas are lacking, we assessed interobserver agreement among practicing expert endocrine pathologists through virtual microscopy in a large multicenter, multinational cohort of genetically well-characterized tumors. Accordingly, we examined the validity of SDHB/SDHA immunohistochemistry to identify patients with succinate dehydrogenase-related pheochromocytomas/paragangliomas and of SDHB immunohistochemistry as a marker of malignancy.

\section{Materials and methods}

\section{Case Selection}

A total of 351 paraganglionic tumors from 333 patients of median age 46 years (ranging from 5.5 to 84 years; $56 \%$ females) were retrieved from 15 specialized centers from Europe, United States, and Australia: (1) Université catholique de Louvain, Brussels, Belgium (95 samples from 84 patients), (2) Hôpital Européen Georges Pompidou, Paris, France (68 samples from 67 patients), (3) University of Florence, Florence, Italy (40 samples), (4) National Institutes of Health (NIH), Bethesda, MD, USA (24 samples), (5) Klinikum der Universität München, Munich, Germany (20 samples), (6) Radboud University Nijmegen Medical Center, Nijmegen, The Netherlands (18 samples from 17 patients), (7) Instituto Português de Oncologia de Lisboa Francisco Gentil E.P.E., Lisbon, Portugal (15 samples from 12 patients), (8) Hôpital Cochin, Paris, France (13 samples), (9) Jagiellonian University Medical College, Krakow, Poland (12 samples), (10) Technische Universität Dresden, Dresden, Germany (11 samples), (11) San Luigi Gonzaga Hospital and University of Turin, Turin, Italy (11 samples), (12) Erasmus MC Cancer Institute, Rotterdam, The Netherlands (10 samples from 8 patients), (13) University of Sydney, Sydney, Australia (8 samples), (14) Spanish National Cancer Research Centre (CNIO), Madrid, Spain (5 samples), and (15) Hospital Universitario San Cecilio, Granada, Spain (1 sample). Clinical and genetic characteristics of these patients are detailed in Supplementary Tables 1 and 2. Thirty samples (30 out of $351 ; 8.54 \%$ ) were considered malignant (Supplementary Table 2) as primary tumors and/or recurrences in the presence of metastatic disease to sites where chromaffin tissue is not normally found ${ }^{4}$ or as metastases themselves.

Out of 351 tumor samples, (1) 73 were $S D H-x$ mutated (39SDHD, $24 S D H B, 4 S D H A, 4$ SDHAF2, and $2 S D H C$ ), (2) 105 non-SDH-X mutated (37 VHL, 25 RET, 21 NF1, 8 MAX, 6 HIF2A, 4 TMEM127, and 4 HRAS), (3) 128 wild-type cases (7 head and neck paragangliomas, 13 extra-adrenal paragangliomas, and 108 pheochromocytomas) that have been tested negative for mutations and large deletions in the $S D H-x$ genes, and (4) 45 samples with incomplete $S D H-x$ molecular genetic analysis in terms of either $S D H-x$ genes or the techniques performed, that is, Sanger sequencing and/or multiplex ligation-dependent probe amplification. A total of 225 samples were analyzed at least for 3 pheochromocytoma/ paraganglioma susceptibility genes with 129 and 30 harboring mutations at the germline and somatic level, respectively (Supplementary Table 1). Based on clinical grounds, 19 tumors were considered $N F 1$, RET, or VHL mutated (Supplementary Table 1).

None of these tumor samples have been previously published elsewhere in terms of SDHB/SDHA immunohistochemical investigation and all were anonymously assessed according to the Proper Secondary Use of Human Tissue code established by the Dutch Federation of Medical Scientific Societies (http://www.federa.org). Informed consent was obtained for genetic analysis and access to the clinical data in accordance with institutional 
guidelines. The Medical Ethical Committee of the Erasmus MC approved the study.

\section{SDHB/SDHA Immunohistochemistry}

Each case was thoroughly reviewed and representative unstained glass slide(s) $(n=147)$ and/or formalin-fixed, paraffin-embedded block(s) $(n=204)$ were selected and further provided for immunohistochemical analysis within a single research setting (Department of Pathology, Erasmus MC Cancer Institute, Rotterdam, The Netherlands) with the following protocol. Slides and formalin-fixed, paraffinembedded whole-tissue sections of $4 \mu \mathrm{m}$ thickness were stained with commercially available antibodies: (1) mouse monoclonal Ab14715 antibody (Mitosciences, Abcam, Cambridge, UK; 1:500 dilution) against SDHA and (2) rabbit polyclonal HPA002868 antibody (Sigma-Aldrich, St Louis, MO, USA; 1:400 dilution) against SDHB on an automatic Ventana Benchmark Ultra System (Ventana Medical Systems, Tuscon, AZ, USA) using Ultraview DAB detection system preceded by heat-induced epitope retrieval with Ventana Cell Conditioning $1\left(\mathrm{pH}\right.$ 8.4) at $97^{\circ} \mathrm{C}$ for 52 and $92 \mathrm{~min}$, respectively. Diaminobenzidine was used as the chromogen.

\section{Telepathology Application}

High-resolution, whole-slide images were acquired from 702 SDHB/SDHA immunostainings using a NanoZoomer Digital Pathology System (Hamamatsu Photonics KK, Japan) working at a resolution of $0.23 \mu \mathrm{m} /$ pixel. The immunostainings were scanned at $\times 40$ magnification and automatically digitized in their proprietary NanoZoomer Digital Pathology Image file format. A quality control was subsequently set to ensure good focus. Between August 2012 and December 2013, digital files were consecutively uploaded in six sets to a server at Erasmus MC through the standard File transfer Protocol with URL http:// digimic.erasmusmc.nl/, enabling online worldwide viewing through a virtual microscopy interface (NanoZoomer Digital Pathology.view Viewer Software, Hamamatsu Photonics KK).

\section{Participants and Interpretation of Staining Results}

Seven pathologists, including five who had published on SDHB and/or SDHA immunohistochemical assessments and two who had dealt with endocrine pathology on diagnostic and research grounds for many years (AJG, F van N, AST, FT, MV, XM-G, and RRdeK), received: (1) a word file detailing the context and the objectives of the project along with an instructory panel of SDHB/SDHA immunohistochemistry, (2) a Virtual Microscopy (NanoZoomer Digital Pathology) Manual, (3) the corresponding link providing access to the virtual slides of the first set of tumors, and (4) a scoring list to be completed during SDHB/SDHA immunohistochemical evaluations.

All virtual slides were distributed online, reviewed by each observer in a blinded manner without knowledge of the corresponding clinicopathological and genetic data or scores assigned by other pathologists and scored as follows: (1) with regard to SDHB immunohistochemistry: Positive as granular cytoplasmic staining displaying the same intensity as internal positive control (endothelial cells, sustentacular cells, lymphocytes); Negative as completely absent staining in the presence of an internal positive control; Weak diffuse as a cytoplasmic blush lacking definite granularity contrasting the strong granular staining of internal positive control; Heterogeneous as granular cytoplasmic staining combined with a cytoplasmic blush lacking definite granularity or completely absent staining in the presence of an internal positive control throughout the same slide; Noninformative as completely absent staining in the absence of an internal positive control; and (2) with regard to SDHA immunohistochemistry: Positive as granular cytoplasmic staining displaying the same intensity as internal positive control (endothelial cells, sustentacular cells, lymphocytes); Negative as completely absent staining in the presence of an internal positive control; Heterogeneous as granular cytoplasmic staining combined with a cytoplasmic blush lacking definite granularity or completely absent staining in the presence of an internal positive control throughout the same slide; Noninformative as completely absent staining in the absence of an internal positive control.

In an effort to simulate widespread adoption of the scoring system as would occur in community practice, no prescoring consensus meeting was organized. In order to imitate clinical practice as much as possible for SDHB/SDHA immunohistochemical interpretations, we selected a large retrospective cohort comprising $\mathrm{SDH}-\mathrm{X}^{-}$and non$S D H$-X-mutated paraganglionic tumors with and without mutations in the remainder pheochromocytoma/paraganglioma-associated genes.

\section{Statistical Analysis}

Interobserver agreement was assessed using $\kappa$ statistics; the strength of the former was evaluated with criteria previously described by Landis and Koch. ${ }^{47}$ A $\kappa$-value of $<0$ indicates less than chance agreement, $<0.20$ is regarded as slight agreement, $0.21-0.40$ as fair agreement, $0.41-0.60$ as moderate agreement, $0.61-0.80$ as substantial agreement, $0.81-0.99$ as almost perfect agreement, and 1 indicates perfect agreement. A dichotomous classification was used for the analysis of the pathologists' evaluations (negative/weak diffuse and positive) as well as a three-tiered classification approach (negative/weak diffuse, positive, and heterogeneous). Consensus was 
Table 1 Interobserver agreement ( $\kappa$-values) for SDHA (upper half) and SDHB (lower half) immunohistochemistry

\begin{tabular}{|c|c|c|c|c|c|c|c|}
\hline & Observer 1 & Observer 2 & Observer 3 & Observer 4 & Observer 5 & Observer 6 & Observer 7 \\
\hline Observer 1 & - & 0.7471 & 0.7471 & 0.4942 & 0.5944 & 0.8557 & 0.8557 \\
\hline Observer 2 & 0.7623 & - & 0.7471 & 0.4942 & 0.7972 & 1.0000 & 0.8557 \\
\hline Observer 3 & 0.8561 & 0.8593 & - & 0.4942 & 0.5387 & 0.8557 & 0.8557 \\
\hline Observer 4 & 0.6282 & 0.6508 & 0.6819 & - & 0.3542 & 0.5672 & 0.5672 \\
\hline Observer 5 & 0.7943 & 0.7998 & 0.8286 & 0.5981 & - & 0.6628 & 0.6628 \\
\hline Observer 6 & 0.7199 & 0.8021 & 0.7721 & 0.7276 & 0.7759 & - & 1.0000 \\
\hline Observer 7 & 0.8733 & 0.6476 & 0.7923 & 0.5318 & 0.6880 & 0.6621 & - \\
\hline
\end{tabular}

All agreements $P<0.0001$.

defined as agreement at least among five out of seven pathologists reaching the same interpretation on positive, negative/weak diffuse, heterogeneous, and noninformative expression for SDHB/SDHA immunohistochemistry. Discordant evaluation was defined as at least three observers reporting different SDHB/SDHA expression patterns on the same slide. In order to capture the performance of SDHB immunohistochemistry as a predictive tool, we calculated Youden's J statistic (Youden's index) per pathologist either in tumors harboring $S D H-x$ mutations vs non-SDH-x mutations or in $S D H$ - $x$-mutated tumors vs counterparts without identified $S D H-x$ mutations. We used Pearson's $\chi^{2}$ test to associate (1) SDHB IHC status with biological behavior (ie, benignancy vs malignancy) taking into consideration only concordant cases as well as excluding metastases $(n=7)$ and doubled samples $(n=6)$ (Supplementary Table 2), and (2) SDHD mutations and weak diffuse pattern on SDHB immunohistochemistry based on a consolidated call from at least four observers. Two-sided $P$-values of $<0.05$ were considered statistically significant. Statistical analyses were performed using Analyse-it v2.26 (Analyse-it Software, Leeds, UK).

\section{Results}

The interobserver agreement following a two-tiered classification approach (ie, positive and weak diffuse/negative) ranged from moderate to almost perfect for SDHB immunohistochemistry and from fair to perfect for SDHA immunohistochemistry (Table 1). With regard to SDHB immunohistochemistry, the highest agreement was reached between observers 2 and $3(\kappa=0.8593)$ and the lowest between observers 4 and $7 \quad(\kappa=0.5318)$, whereas regarding SDHA immunohistochemistry, the highest agreement was reached between observers 6 and $2 / 7$ $(\kappa=1.0000)$ and the lowest between observers 4 and 5 $(\kappa=0.3542)$. All agreements were highly significant $(P<0.0001)$. Substantial agreement among all the reviewers was observed either with a two-tiered classification (SDHB $\kappa=0.7338$; SDHA $\kappa=0.6707$ ) or a three-tiered classification approach (SDHB $\kappa=0.6543$; SDHA $\kappa=0.7516$ ). Notably, observer 1 as well as observers 3/4/5 did not score any slide as heterogeneous pattern for SDHB and SDHA immunohistochemistry respectively.

Consensus among pathologists was achieved in 348 cases $(99.15 \%)$ for SDHA immunohistochemistry and in 315 cases (89.74\%) for SDHB immunohistochemistry, respectively. Out of 69 tumor samples with $S D H B / S D H C / S D H D / S D H A F 2$ mutations, $62(89.85 \%)$ displayed SDHB immunonegativity and SDHA immunopositivity, whereas 3 of 4 with SDHA mutations $(75 \%)$ showed loss of SDHA/SDHB protein expression (Figure 1). Two SDHD-mutated extra-adrenal paragangliomas (c.274G $>$ T p.Asp92Tyr and c.405delC p.Phe136Leufs*32) were scored as SDHB immunopositive by 5 observers and as immunonegative (weak diffuse) by the other observers (observers 2/5).

All tumors harboring RET, TMEM127, HIF2A, and HRAS mutations, 31 of 37 VHL-mutated tumors (83.7\%), and 20 of 21 NF1 mutated-tumors (95.2\%) displayed retention of SDHB/SDHA expression (Figure 2). Six benign VHL-mutated pheochromocytomas ( 6 out of $37 ; \sim 16 \%$ ) and one malignant NF1mutated extra-adrenal paraganglioma (1 out of 21 ; $\sim 5 \%)$ were evaluated as SDHB immunonegative (VHL: by all observers (3 cases), 6 observers (1 case), and 5 observers (2 cases); NF1: by 6 observers (1 case)) in the absence of $S D H-x$ mutations in four of these cases (two examined at the germline, one at the germline and somatic, and one at the somatic level). Data on the exact mutations were available only in four cases (VHL p.Ser80Asn, p.Arg161*, p.Arg167Gln, and NF1 p.Trp561*).

In the absence of $S D H-x$ mutations, 119 out of 128 paraganglionic tumors (93\%) were scored as SDHB/ SDHA immunopositive, whereas the remainder (9 out of $128 ; 7 \%$ ) as SDHB immunonegative/SDHA immunopositive. Clinicopathological and genetic data of the latter from four independent centers are detailed in Table 2.

Discordant evaluations of SDHB immunohistochemistry were reported in 5 tumors endowed with $S D H-x$ (SDHD/SDHB/SDHAF2) mutations, $11 \mathrm{VHL}-$ and 2 RET-mutated tumors, as well as 18 tumors without identified $S D H-x$ mutations, whereas of SDHA immunohistochemistry concerned $2 S D H$-x-mutated tumors (SDHA-/SDHD-) and 1 NF1-mutated tumor. 

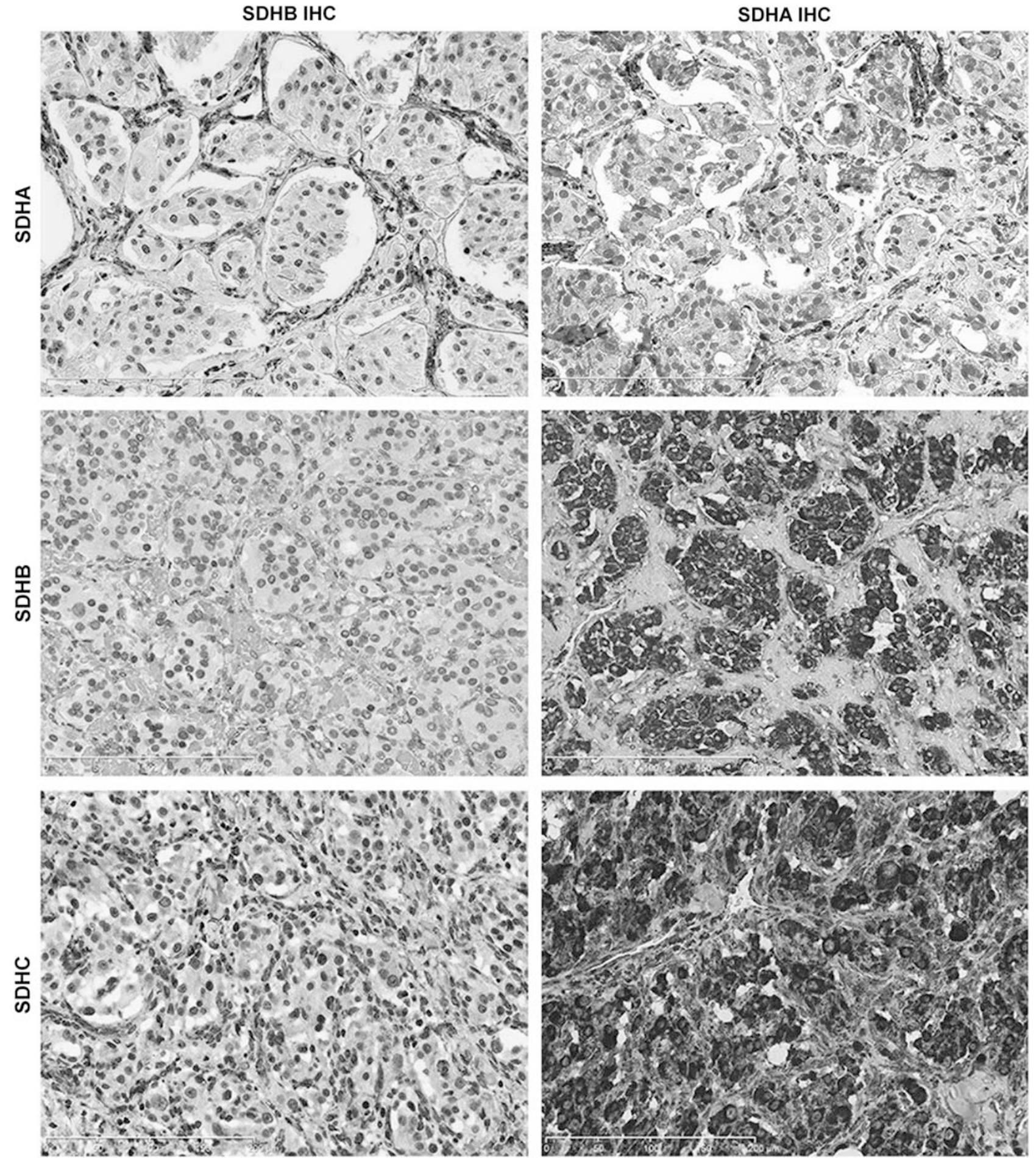

Figure 1 SDHA and SDHB immunohistochemistry in pheochromocytomas/paragangliomas endowed either with SDHA germline mutation displaying loss of SDHA/SDHB protein expression or with SDHB, SDHC, SDHD, and SDHAF2 germline mutations exhibiting loss of SDHB, but intact SDHA expression. Note the granular, cytoplasmic staining for SDHA/SDHB in normal cells of the intratumoral fibrovascular network that serve as internal positive controls.

The classification of stainings as 'noninformative' and 'heterogeneous' represented the major reason for SDHB/SDHA immunohistochemical discrepancies in the $S D H$-X-mutated subgroup, whereas the 'weak diffuse' category accounted largely for those in the $S D H$ - $x$-wild-type and $V H L$-mutated subsets.

The association between the predicted SDH genetic status and SDHB immunohistochemistry 

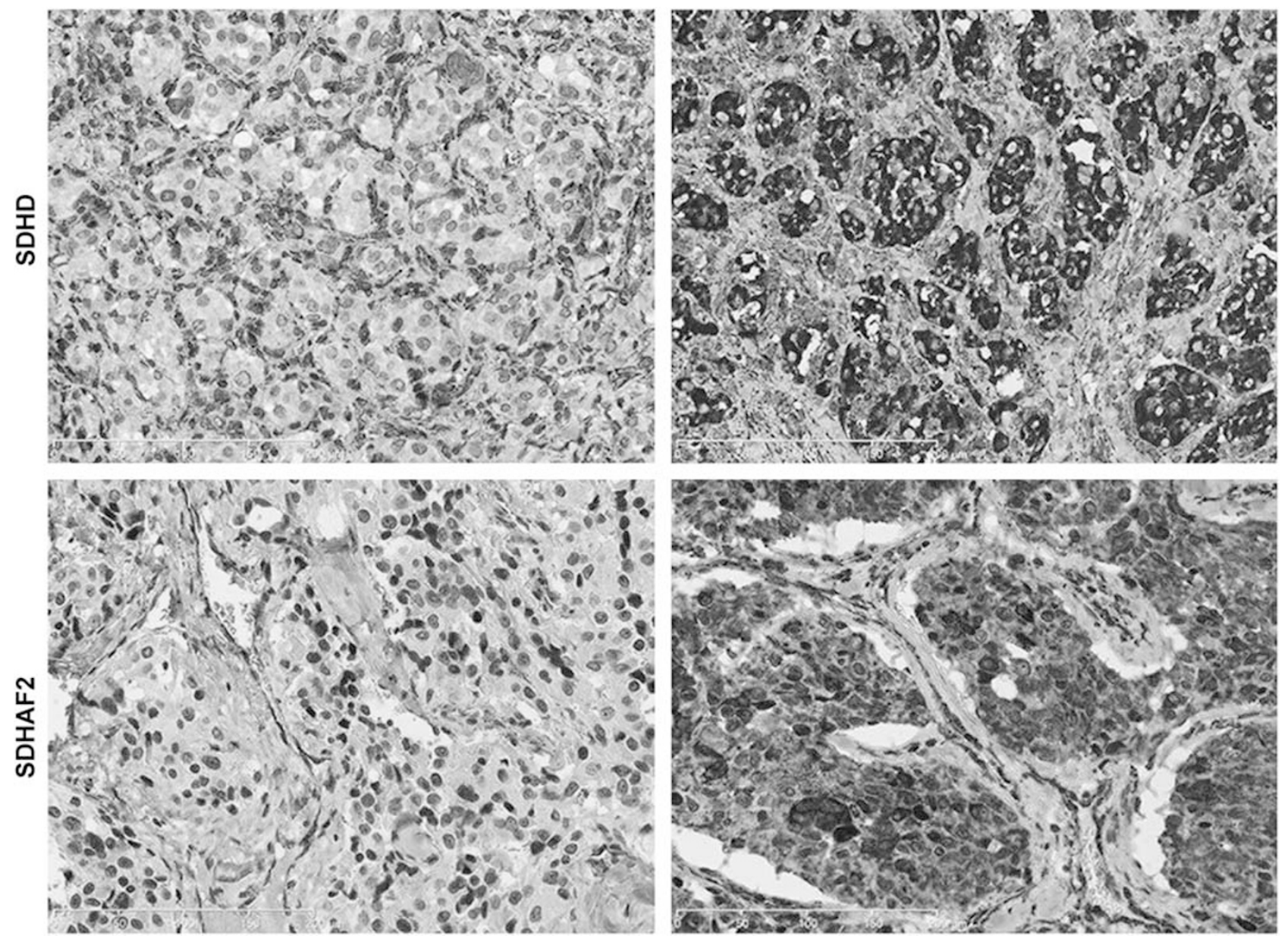

Figure 1 Continued.

was investigated for each observer. The sensitivity of this approach, defined as the percentage of $S D H-x$ mutated tumors that are SDHB immunonegative, ranged from 83.58 to $98.57 \%$ (mean $94.23 \%$ ). The specificity, defined as the percentage of either non$S D H$-x-mutated tumors or tumors without identified $S D H-x$ mutations that are SDHB immunopositive, varied between 74.03 and $96.11 \%$ (mean $84.35 \%$ ) as well as 83.06 and $92.91 \%$ (mean $86.67 \%$ ), respectively. Observer 1 was the best predictor with a Youden's index of 0.880 and 0.860 (Table 3). A significant correlation was observed between SDHB immunonegativity and malignancy $(P=0.00019)$. No association could be shown between the $S D H D$ mutations and the weak diffuse pattern on SDHB immunohistochemistry $(P=0.1490)$.

\section{Discussion}

Immunohistochemistry has revolutionized the practice of endocrine pathology during the last decade. In parallel with recent advances in molecular genetics, immunohistochemistry has been shown to detect various types of molecular alterations, that is, $B R A F$ V600E mutation in papillary thyroid carcinomas, ${ }^{48}$ PTEN mutations in various neoplastic thyroid lesions, ${ }^{49}$ CTNNB1 mutations in cribriform-morular variant of papillary thyroid carcinoma, undifferentiated carcinomas of the thyroid gland and adrenocortical carcinomas, ${ }^{48,50,51}$ TP53 mutations as well as mutations in mismatch repair (MMR) genes such as MLH1, MSH2, MSH6, and PMS2 in adrenocortical carcinomas, ${ }^{51-53}$ HRPT2 mutations in parathyroid carcinomas and hyperparathyroidism-jaw tumor syndrome-related adenomas, ${ }^{48,54}$ PRKAR1A mutations in Carney complex-associated tumors, ${ }^{55-57}$ and $S D H-, F H$ - as well as MAX deleterious-mutations in pheochromocytomas/paragangliomas. ${ }^{8,10,11,58,59}$

Loss of SDHB protein expression is seen in pheochromocytomas/paragangliomas either harboring a mutation in any of the $S D H$ genes or with somatic hypermethylation of the SDHC promoter region, ${ }^{42}$ whereas loss of both SDHB and SDHA immunoreactivity is demonstrated only in the context of an SDHA mutation. ${ }^{8-20}$ In agreement with previous studies, ${ }^{8,10,11,17-20} S D H B-/ C$ - $/ D$ - and $S D H A$ mutated tumors displayed the aforementioned 


\section{SDHB IHC}
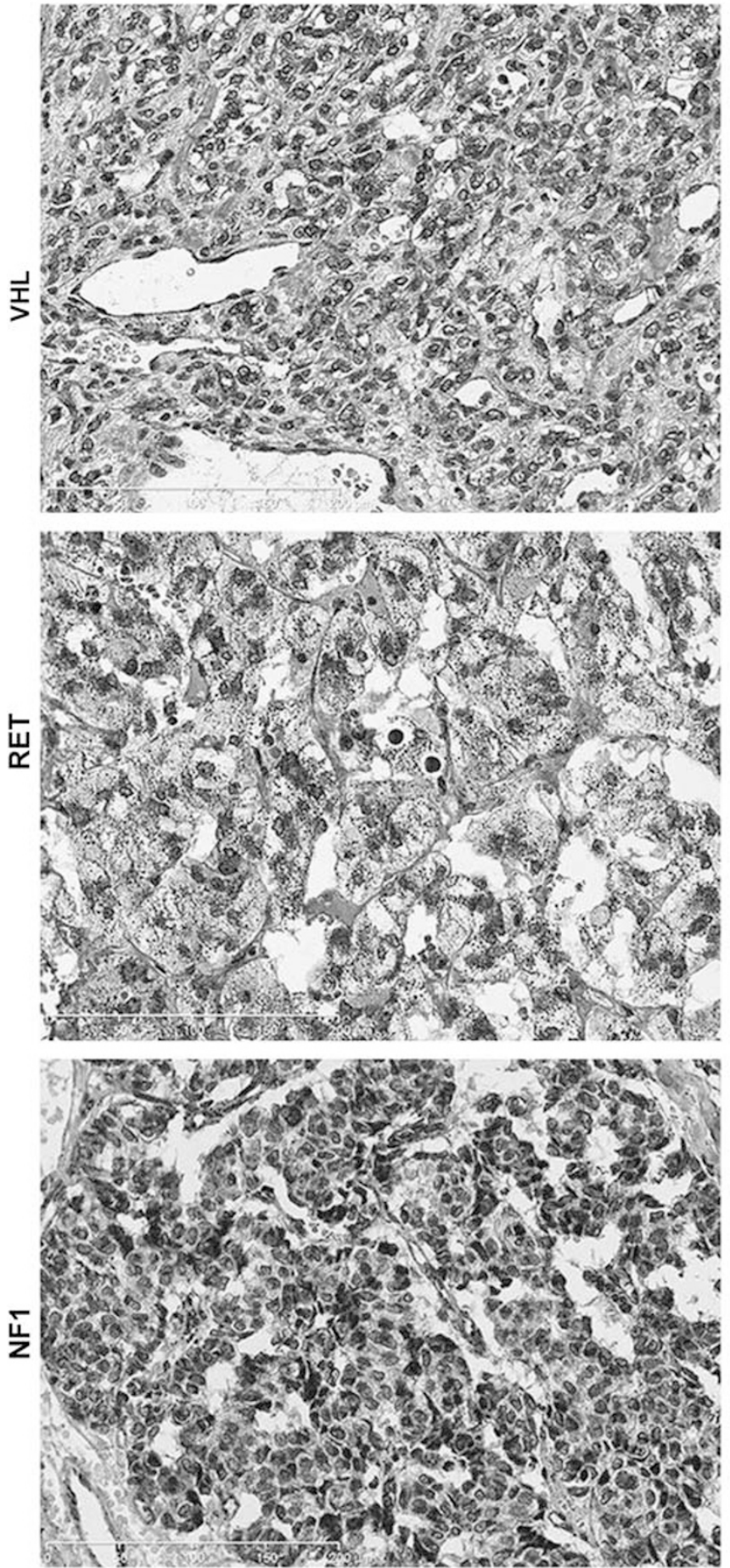

SDHA IHC
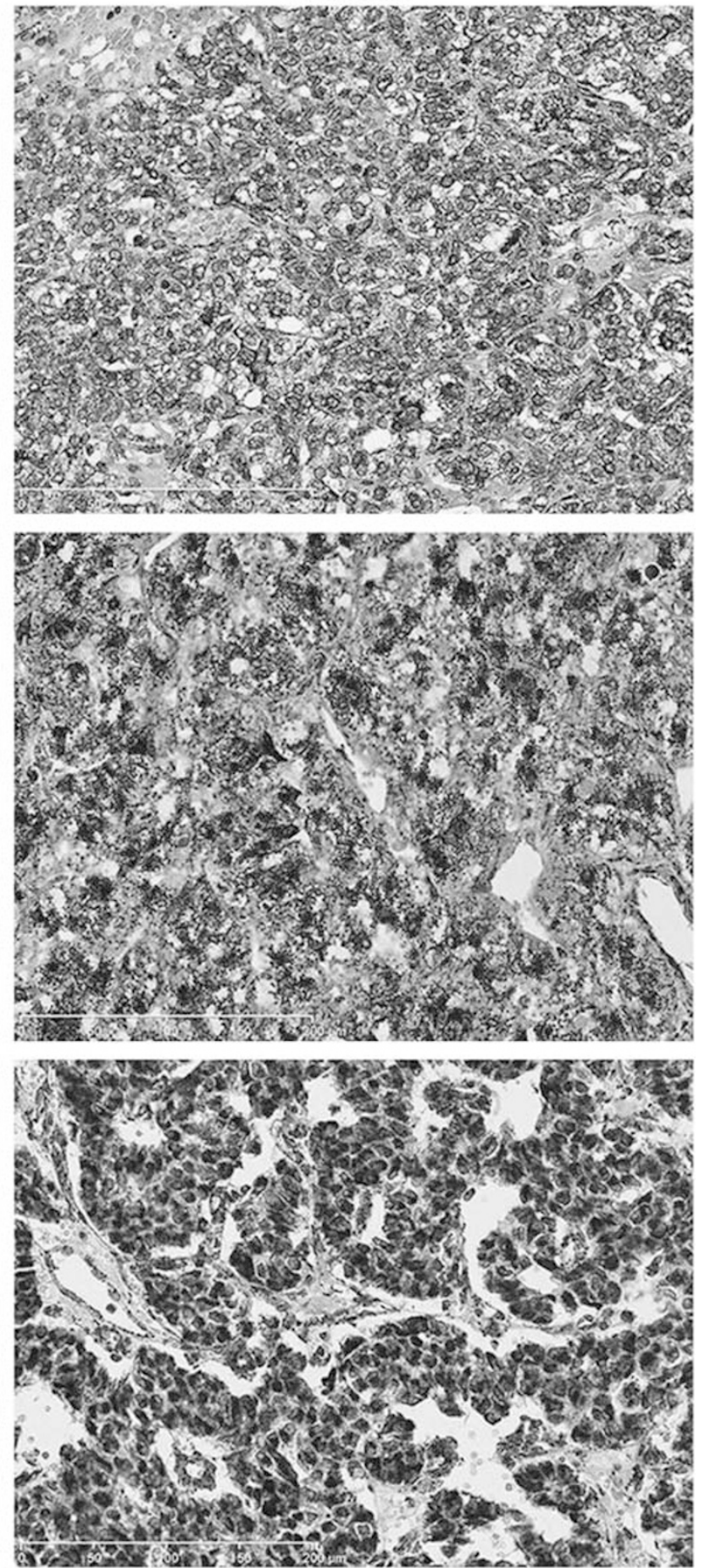

Figure 2 Intact SDHB and SDHA protein expression in non-SDH-X-mutated paraganglionic tumors harboring germline or somatic $V H L$, RET, NF1, TMEM127, MAX, EPAS1, and HRAS mutations. Note the granular, cytoplasmic staining for SDHA/SDHB in normal cells of the intratumoral fibrovascular network that serve as internal positive controls.

immunoexpression patterns with SDHAF2-mutated counterparts showing SDHB immunonegativity and SDHA immunopositivity. Notably, all tumors harboring RET, TMEM127, HIF2A, and HRAS mutations displayed retention of SDHB/SDHA expression, whereas six benign VHL-mutated pheochromocytomas and one malignant NF1-mutated extra-adrenal paraganglioma were evaluated as SDHB immunonegative. The latter contrasts previous observations in 37 pheochromocytomas/paragangliomas 

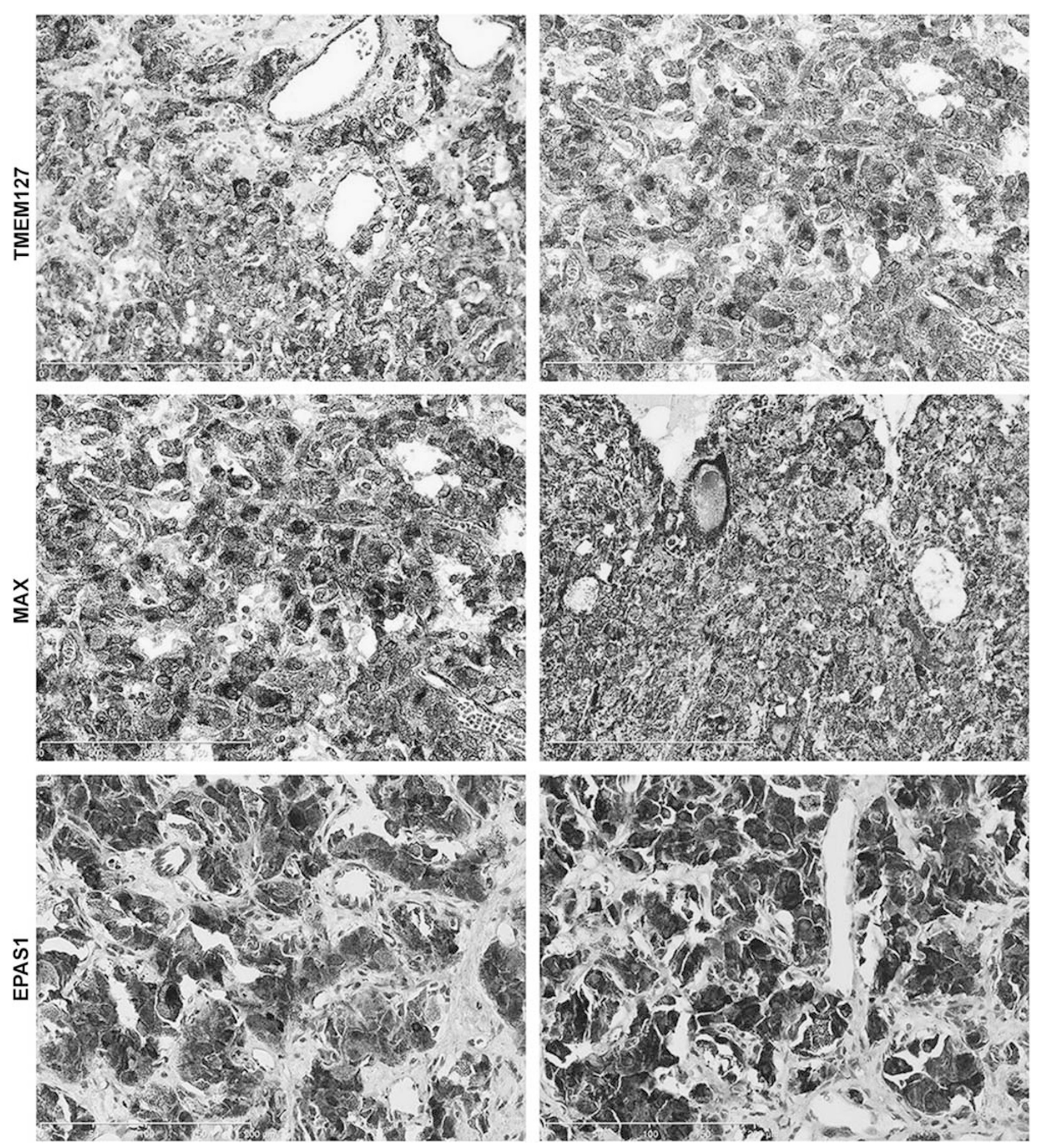

Figure 2 Continued.

and 14 pheochromocytomas endowed with $V H L^{8,11}$ and NF1 mutations, ${ }^{8,10}$ respectively. By using a mouse monoclonal (21A11) SDHB antibody at a low concentration (1 in 1000), Gill et al ${ }^{10}$ suggested that VHL-associated tumors could be classified as negative or weak diffuse rather than positive as demonstrated by a high concentration approach of two SDHB antibodies. ${ }^{8}$ In accordance, loss of SDHB protein expression has been recently displayed in a subset of NF1-mutated paraganglionic tumors (J Favier 2014, personal communication). The remote possibility of a double mutant, potentially explaining the SDHB immunonegativity by an additional $S D H-x$ mutation, was ruled out in four of these seven cases occurring in the VHL- and NF1-deficient setting.

To further expand earlier observations, ${ }^{8,11} 9$ of 128 $(7 \%)$ tumors without identified $S D H-x$ mutations 

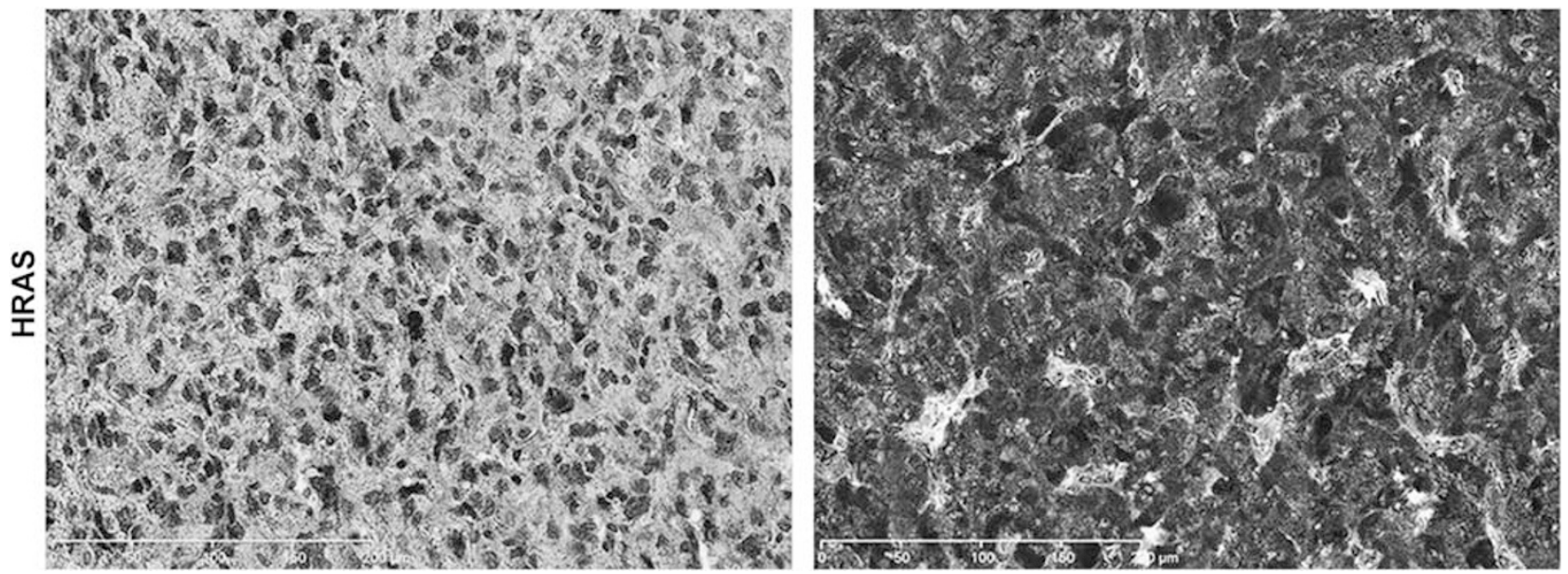

Figure 2 Continued.

Table 2 Clinicopathological and genetic data of patients with SDHB-immunonegative paraganglionic tumors in the absence of $S D H-x$ mutations

\begin{tabular}{|c|c|c|c|c|c|c|c|c|c|c|c|c|c|c|c|}
\hline \multirow[b]{2}{*}{$\begin{array}{l}\text { Sample } \\
\text { code }\end{array}$} & \multicolumn{3}{|c|}{ Syndromic presentation } & \multirow[b]{2}{*}{ Sex } & \multirow[b]{2}{*}{$\begin{array}{l}\text { Tumor } \\
\text { type }\end{array}$} & \multirow[b]{2}{*}{ Dignity } & \multicolumn{8}{|c|}{ Molecular genetic testing of PCC/PGL susceptibility genes a } & \multirow[b]{2}{*}{$M A X$} \\
\hline & $\begin{array}{c}\text { Familial } \\
\text { PCC/ } \\
\text { PGL history }\end{array}$ & $\begin{array}{c}\text { Multiple } \\
\text { tumors }\end{array}$ & $\begin{array}{c}\text { Age at } \\
\text { presentation }\end{array}$ & & & & $S D H B$ & $S D H D$ & $S D H C$ & $S D H A$ & SDHAF2 & SDHAF1 & VHL & TMEM127 & \\
\hline BEL 30 & No & No & 43 & $\mathrm{~F}$ & HN PGL & B & - & - & - & - & - & ND & - & - & - \\
\hline BEL 67 & No & No & 36 & $\mathrm{M}$ & HN PGL & B & - & - & - & - & - & ND & - & - & - \\
\hline DR 11 & No & No & 27 & $\mathrm{~F}$ & HN PGL & B & - & - & - & ND & - & ND & ND & - & - \\
\hline ITA 28 & No & No & 73 & $\mathrm{~F}$ & HN PGL & B & - & - & - & - & - & ND & - & - & - \\
\hline DR $10^{\mathrm{b}}$ & No & Yes & 33 & $\mathrm{~F}$ & EA PGL & B & - & - & - & - & - & - & ND & ND & - \\
\hline BEL 66 & No & No & 15 & $\mathrm{~F}$ & EA PGL & $\mathrm{M}$ & - & - & - & - & - & ND & - & - & - \\
\hline BEL 116 & No & No & 20 & M & PCC & $\mathrm{B}$ & - & - & - & - & - & ND & - & - & ND \\
\hline ITA 48 & No & No & 47 & $\mathrm{~F}$ & PCC & B & - & - & - & - & - & ND & - & - & - \\
\hline FR115 ${ }^{\mathrm{C}}$ & No & No & 23 & $\mathrm{M}$ & PCC & B & - & - & - & - & ND & ND & - & - & - \\
\hline
\end{tabular}

Abbreviations: B, benign; EA, extra-adrenal; F, female; HN, head and neck; M, male; M, malignant; ND, not done; PCC, pheochromocytoma; PGL, paraganglioma.

${ }^{\mathrm{a}} \mathrm{SDH}-\mathrm{x}$ genes have been tested both for point mutations and large deletions at the germline level with DR10 and ITA48 also investigated at the somatic level.

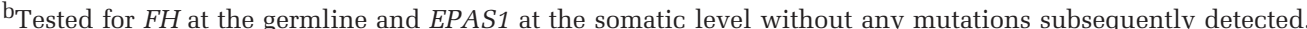

${ }^{\mathrm{C}}$ Tested for RET mutations as well for $S D H-\mathrm{x} / \mathrm{VHL}$ large deletions at the germline level without any mutations subsequently detected.

were evaluated as SDHB immunonegative (Table 2). Van Nederveen et $a l^{8}$ and Castelblanco et $a 1^{11}$ reported on 9 cases ( 6 out of $53 ; 11 \%$ and 3 out of $19 ; 15.7 \%$ ) displaying loss of SDHB expression in the absence of $S D H B, S D H C, S D H D, V H L$, or RET mutation. Nevertheless, these studies lacked either SDHA/SDHAF2 genetic testing 8,11 or screening for large-scale $S D H$-x deletions ${ }^{11}$ that may account for higher percentages. Intriguingly, in the present study, eight SDHB-immunonegative tumors were nonmetastatic in the absence of $S D H-x$ mutations (Table 2), bearing a close resemblance to the Carney triad-associated counterparts in terms of SDHB immunohistochemistry and biologic behavior. ${ }^{4,29,60}$ Because somatic hypermethylation of $S D H C$ was not investigated, the possibility that the aforementioned tumors represented cases of Carney triad could not be assessed. Nevertheless, as shown herein, SDHB immunohistochemical status overall is strongly correlated with the clinical behavior of pheochromocytoma/paraganglioma, further strengthening the role of SDHB immunohistochemistry as a prognostic marker. ${ }^{46,61}$

Our data reinforce the notion that immunohistochemistry is a valid tool to identify patients at risk for familial succinate dehydrogenase-related pheochromocytoma/paraganglioma syndromes, although occasionally this might be difficult even in a specialized setting (Table 3). Exemplifying the latter, two extra-adrenal paragangliomas with missense and frameshift $S D H D$ mutations were scored as SDHB immunopositive by five observers. Similar discrepancy has been previously reported for an extraadrenal paraganglioma harboring a nonsense $S D H D$ mutation (c. $14 \mathrm{G}>\mathrm{A}$ p.Trp5 ${ }^{*}$ ) in a patient with Carney Stratakis syndrome. ${ }^{31}$ Given that the patient additionally developed an SDHB-immunonegative gastrointestinal stromal tumor ${ }^{31}$ and that identical 
Table 3 Associating predicted SDHB IHC status either with $S D H$ - $x$-mutated vs non-SDH-X-mutated status (A) or with $S D H$ - $x$-mutated vs $S D H$ - $x$-wild-type status (B) ${ }^{\mathrm{a}}$

\begin{tabular}{|c|c|c|c|c|c|c|c|}
\hline & Observer 1 & Observer 2 & Observer 3 & Observer 4 & Observer 5 & Observer 6 & Observer 7 \\
\hline \multicolumn{8}{|l|}{$A$} \\
\hline Sensitivity & $95.71 \%$ & $98.57 \%$ & $94.44 \%$ & $93.22 \%$ & $98.57 \%$ & $95.52 \%$ & $83.58 \%$ \\
\hline Specificity & $92.30 \%$ & $77.66 \%$ & $90.00 \%$ & $74.03 \%$ & $82.35 \%$ & $78.02 \%$ & $96.11 \%$ \\
\hline PPV & $89.33 \%$ & $75.00 \%$ & $87.17 \%$ & $67.07 \%$ & $79.31 \%$ & $76.19 \%$ & $93.33 \%$ \\
\hline NPV & $96.96 \%$ & $98.76 \%$ & $95.74 \%$ & $95.06 \%$ & $98.82 \%$ & $95.94 \%$ & $90.00 \%$ \\
\hline Pval & $P<0.0001$ & $P<0.0001$ & $P<0.0001$ & $P<0.0001$ & $P<0.0001$ & $P<0.0001$ & $P<0.0001$ \\
\hline Youden's index & 0.880 & 0.762 & 0.844 & 0.672 & 0.809 & 0.735 & 0.796 \\
\hline \multicolumn{8}{|l|}{$B$} \\
\hline Sensitivity & $95.71 \%$ & $98.57 \%$ & $94.44 \%$ & $93.22 \%$ & $98.57 \%$ & $95.52 \%$ & $83.58 \%$ \\
\hline Specificity & $90.47 \%$ & $83.06 \%$ & $87.70 \%$ & $84.55 \%$ & $83.73 \%$ & $84.21 \%$ & $92.91 \%$ \\
\hline PPV & $84.81 \%$ & $76.66 \%$ & $81.92 \%$ & $74.32 \%$ & $77.52 \%$ & $78.04 \%$ & $86.15 \%$ \\
\hline NPV & $97.43 \%$ & $99.03 \%$ & $96.39 \%$ & $96.29 \%$ & $99.03 \%$ & $96.96 \%$ & $91.47 \%$ \\
\hline Pval & $P<0.0001$ & $P<0.0001$ & $P<0.0001$ & $P<0.0001$ & $P<0.0001$ & $P<0.0001$ & $P<0.0001$ \\
\hline Youden's index & 0.860 & 0.816 & 0.821 & 0.777 & 0.823 & 0.797 & 0.764 \\
\hline
\end{tabular}

Abbreviations: Pval, $P$-value $\chi^{2}$ test; PPV, positive predictive value; NPV, negative predictive value.

Youden's index is defined as sensitivity+specificity-1. The higher the Youden's index, the better the prediction.

Sensitivity is defined as the percentage of SDH-x-mutated tumors that are SDHB immunonegative Specificity is defined as the percentage of non$S D H-x$-mutated tumors or tumors without identified SDH-x mutations that are SDHB immunopositive.

${ }^{\mathrm{a}}$ Heterogeneous and noninformative scorings are excluded.

missense and nonsense $S D H D$ mutations in other tumors have led to absence of SDHB expression, ${ }^{5,8}$ it is possible that either the second hit in the $S D H D$ gene in the paraganglioma resulted in an inactive succinate dehydrogenase complex with preservation of antigenicity or that the interpretation was erroneous. Of note, every pathologist in the current study missed at least one $S D H$ - $x$-related tumor, and these most frequently involved mutations in $S D H D$. This suggests SDHD immunohistochemistry as a potential complementary tool to SDHB immunohistochemistry to identify $S D H D$-mutated patients. ${ }^{62}$ Further adding to those rare familial cases characterized by disparity between molecular genetic aberrations of a tumor suppressor gene and retention of protein expression, ${ }^{63}$ one papillary renal cell carcinomas arising in a patient with a germline missense $S D H C$ mutation (c.3G $>$ A p.M1I) and harboring somatic loss of heterozygosity of the SDHC locus paradoxically displayed SDHB immunopositivity. ${ }^{36}$ Taken together, SDHB immunohistochemistry and $S D H-x$ genetic analysis should be viewed as complementary tests. In cases of strong clinical suspicion, follow-up mutational analysis should be considered despite retention of SDHB expression.

The good level of reproducibility in the current study may either reflect a high level of experience with scoring SDHB/SDHA immunostainings among expert endocrine pathologists or be attributable in part to the fact that very precise scoring guidelines were provided. Accordingly, it would be essential to provide such guidelines in clinical reporting templates $^{64}$ as well as to guide development of algorithms for computer-assisted diagnostics in a digital pathology perspective. The classification of stainings as 'non-informative' and 'heterogeneous' represented the major reason for SDHA/SDHB immunohistochemical discrepancies in the $S D H-X^{-}$ mutated subgroup, whereas the 'weak diffuse' category accounted largely for inconsistencies in the $S D H$ - $X$-wild-type and VHL-mutated subsets. These could be potentially ascribed to (1) technical variability owing to differences in fixation time, buffered formalin concentrations, and/or age of the formalinfixed, paraffin-embedded blocks, ${ }^{10,11}$ (2) biological variability, for example, reduced SDHB protein levels in VHL-mutated paraganglionic tumors, ${ }^{65}$ or even to (3-4) individual conceptions and experience from specific staining protocols, as has been shown with immunohistochemistry for MMR proteins. ${ }^{66}$ Technically suboptimal immunostainings were not unexpectedly encountered given the fact that provided material was derived from several pathology laboratories, each following their own fixation and embedding protocols; highlighting the importance of standardizing preanalytical variables in surgical pathology specimens. ${ }^{67,68}$

In contrast to previous studies ${ }^{10,11}$ indicating a stronger correlation of weak diffuse pattern with $S D H D$ mutations, we could not significantly reinforce this particular association. Moreover, SDHB and/or SDHA immunohistochemistry may not always be an all-or-none phenomenon. In particular, two $S D H A$ - and SDHAF2-mutated tumors displayed a heterogeneous expression pattern (Figures 3 and 4) being consistent with previous observations concerning SDHB immunohistochemistry in a pituitary adenoma harboring an $S D H D$ germline mutation. ${ }^{37}$ Along the same lines, heterogeneous expression patterns have been reported both with MMR protein immunohistochemistry in Lynch syndrome and PTEN immunohistochemistry in Cowden syndrome. ${ }^{49,69,70}$ The biologic nature of heterogeneous tumors in these genetic contexts is currently 

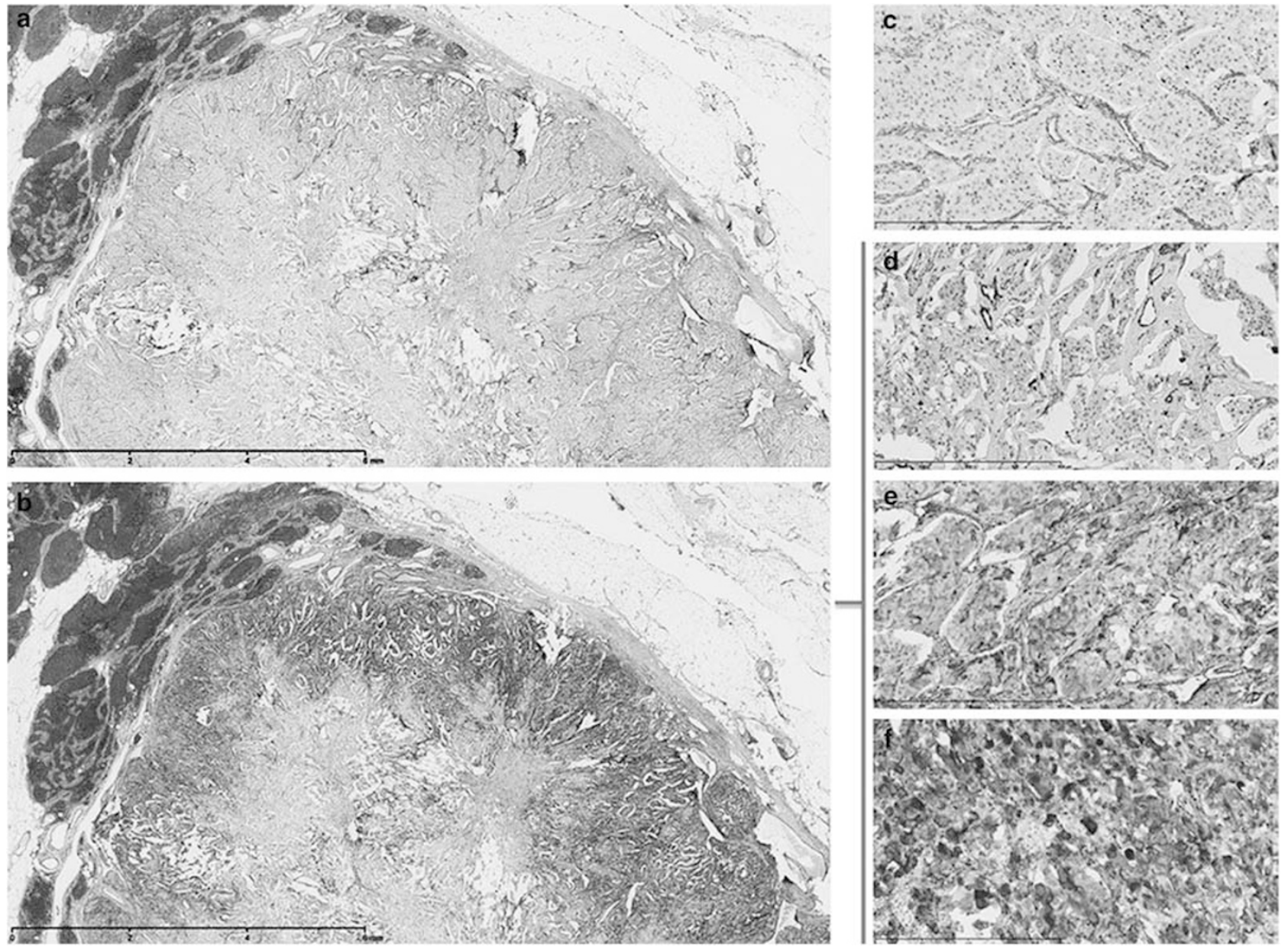

Figure 3 An extra-adrenal paraganglioma harboring an SDHA (c.1534C > T, p.Arg512*) germline mutation, metastatic to a paraaortic lymph node, displaying SDHB immunonegativity (a, c), but a heterogeneous staining pattern for SDHA (b, d-f): central area (d) convincingly negative for SDHA, peripheral areas (f) convincingly positive for SDHA, and transitional zones (e) in between exhibiting cells with intact SDHA expression intermingled with cells with absent SDHA expression. Three pathologists correctly classified this sample as heterogeneous for SDHA, with the remainder four observers as positive for SDHA. Note the granular, cytoplasmic staining for SDHA/ SDHB in normal cells of the intratumoral fibrovascular network that serve as internal positive controls.

unknown. ${ }^{37,49,69,70}$ Because of potential misinterpretation of heterogeneous patterns for SDHB and/or SDHA protein loss, $S D H$ genetic testing is recommended when confronted with such cases.

In addition to a comprehensive next-generation sequencing-based strategy for the analysis of multiple pheochromocytoma/paraganglioma susceptibility genes, ${ }^{43-45}$ several algorithms have been proposed as a targeted approach to genetic testing in clinical practice. ${ }^{8,71-74}$ In this rapidly expanding field, the importance of assessing the pathogenicity of a 'variant of unknown significance' has become a major and complex problem facing diagnostic laboratories. Our data further strengthen the role of SDHB/SDHA immunohistochemistry in determining the functionality of such variants, alone or in an integrated approach with in silico analysis ${ }^{75,76}$ and/or western blot analysis, succinate dehydrogenase enzymatic assay, and mass spectrometric-based measurements of ratios of succinate/fumarate and other metabolites. ${ }^{77-79}$
In the current study, we conclude that SDHB/ SDHA immunohistochemistry represents a reliable tool to identify patients with $S D H-x$ mutations with an additional utility to evaluate the pathogenicity of $S D H$ variants of unknown significance in the new next-generation sequencing era. A heterogeneous SDHB and/or SDHA immunoexpression pattern has to be followed by $S D H$ molecular genetic testing, although a SDHB-immunonegative subset of $V H L$ - and NF1-mutated paraganglionic tumors challenges the issue of specificity for SDHB immunohistochemistry. Hence, if $S D H$ genetics fails to detect a mutation in SDHB-immunonegative tumor, SDHC promoter methylation and/or VHL/NF1 testing with the use of targeted next-generation sequencing is advisable. Our findings highlight the need for quality assessment programs regarding not only standardized staining protocols, but also SDHB/SDHA immunohistochemical evaluation procedures. In a prospective setting, with standardized tissue fixation combined with a locally fine-tuned 

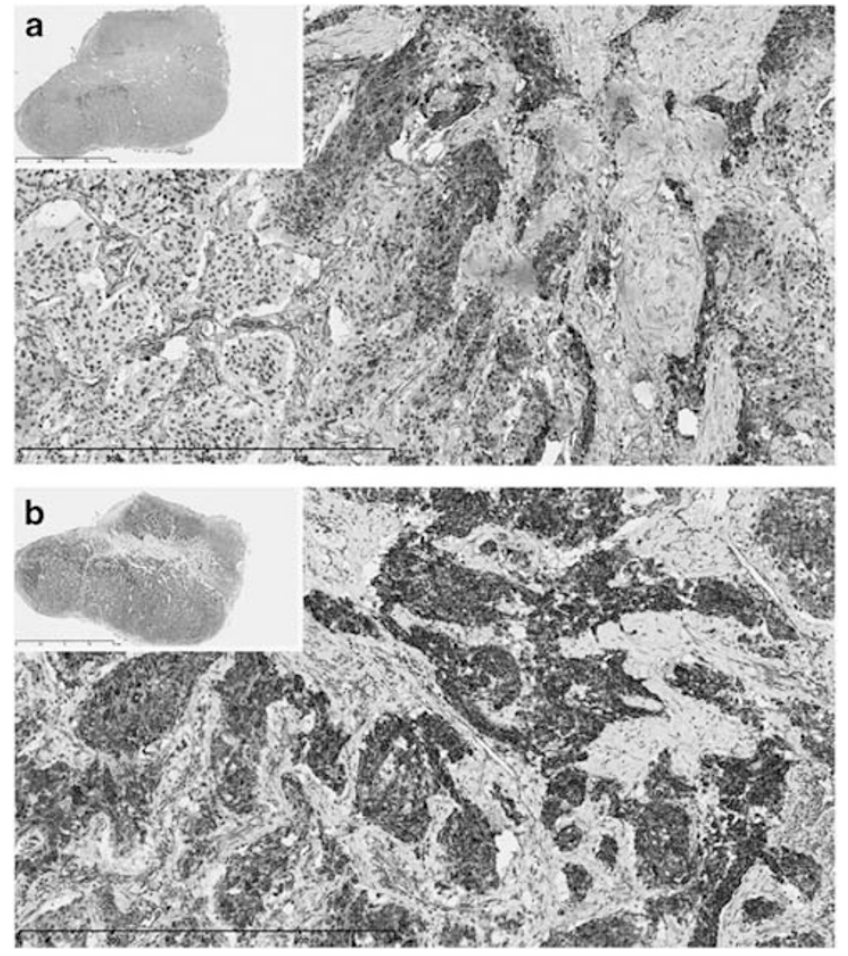

Figure 4 An SDHAF2-mutated (c.232G > A, p.Gly78Arg) head and neck paraganglioma showing areas convincingly negative for SDHB and to a lesser extent areas convincingly positive for SDHB (a). Three pathologists correctly classified this sample as heterogeneous for SDHB, with the remainder four as negative for SDHB, whereas all observers scored it as SDHA immunopositive (b). Note the granular, cytoplasmic staining for SDHB in normal cells of the intratumoral fibrovascular network that serve as internal positive control.

immunohistochemical staining protocol, the sensitivity and specificity of the SDHA/SDHB immunohistochemistry can be improved.

\section{Acknowledgments}

This study was supported by the Seventh Framework Programme (FP7/2007-2013) under grant agreement no. 259735 (ENS@T-Cancer). Genetic analysis of the Belgian subset was partly supported by the Fonds de la Recherche Scientifique Médicale (FRSM) convention number 3.4.587.08 F (to AP). AP, $\mathrm{SA}$, and MV acknowledge the contribution of $\mathrm{N}$ Lannoy, A Mendola, and L Evenepoel (Clin. Univ. St-Luc/UCL) for genetic analysis and F Severino (Clin. Univ. St-Luc) for maintenance of the database. Besides Professor A Mourin (Clin. Univ. St-Luc/ UCL), they are also grateful to all pathologists who contributed tumor samples: Professors $\mathrm{M}$ Delos and B Weynand and Drs M-C Nollevaux and C Fervaille (Cl. Un. De Mont-Godinne, UCL); Drs N Detrembleur, N Blétard, and I Scagnol (CHU Sart-Tilman, Ulg); Drs E Laterre and G Beniuga (IPG Gosselies); Professor H De Raeve and Dr W Jeuris (OLV, Aalst); Dr V Duwel (Ziekenhuis KLINA); Drs A Janssen and S Talpe
(Clniques du Sud-Luxembourg, Arlon); Drs C Robrechts and J Bekaert (Imelda Ziekenhuis); Dr R Duttmann (CHU Brugmann); Dr N de Saint-Aubain (Institut Bordet); and Drs R Achten and K Wouters (Jessa Ziekenhuis). MM, ER, LC, and GN acknowledge the contribution of Dr T Ercolino for the genetic analysis of the Italian (Florence) samples. GE and GB acknowledge the contribution of Drs C Pamporaki, $\mathrm{R}$ Därr, S Richter, and J Brütting for data collection of the German (Dresden) samples. We thank J Shukla (Erasmus MC Cancer Institute) for her valuable technical assistance as well as Drs M Versasky and M Gomez Morales (Hospital Universitario San Cecilio) for providing one SDHD-mutated HNPGL sample.

\section{Disclosure/conflict of interest}

The authors declare no conflict of interest.

\section{References}

1 Tischler AS. Paraganglia In: Mills SE (eds). Histology for Pathologists 4th edn, Lippincott Williams \& Wilkins: Philadelphia, 2012, pp 1277-1299.

2 Dahia PL. Pheochromocytoma and paraganglioma pathogenesis: learning from genetic heterogeneity. Nat Rev Cancer 2014;14:108-119.

3 Yang C, Zhuang Z, Fliedner SM et al. Germ-line PHD1 and PHD2 mutations detected in patients with pheochromocytoma/paraganglioma-polycythemia. J Mol Med (Berl) 2014;93:93-104.

4 Papathomas TG, de Krijger RR, Tischler AS. Paragangliomas: update on differential diagnostic considerations, composite tumors, and recent genetic developments. Semin Diagn Pathol 2013;30:207-223.

5 Papathomas TG, Gaal J, Corssmit EP et al. Nonpheochromocytoma (PCC)/paraganglioma (PGL) tumors in patients with succinate dehydrogenase-related PCCPGL syndromes: a clinicopathological and molecular analysis. Eur J Endocrinol 2013;170:1-12.

6 Belinsky MG, Rink L, von Mehren M. Succinate dehydrogenase deficiency in pediatric and adult gastrointestinal stromal tumors. Front Oncol 2013;3:117.

7 Gill AJ, Hes O, Papathomas T et al. Succinate dehydrogenase (SDH)-deficient renal carcinoma: a morphologically distinct entity: a clinicopathologic series of 36 tumors from 27 patients. Am J Surg Pathol 2014;38:1588-1602.

8 van Nederveen FH, Gaal J, Favier J et al. An immunohistochemical procedure to detect patients with paraganglioma and phaeochromocytoma with germline SDHB, SDHC, or SDHD gene mutations: a retrospective and prospective analysis. Lancet Oncol 2009;10:764-771.

9 Burnichon N, Rohmer V, Amar L et al. The succinate dehydrogenase genetic testing in a large prospective series of patients with paragangliomas. J Clin Endocrinol Metab 2009;94:2817-2827.

10 Gill AJ, Benn DE, Chou A et al. Immunohistochemistry for SDHB triages genetic testing of SDHB, SDHC, and SDHD in paraganglioma-pheochromocytoma syndromes. Hum Pathol 2010;41:805-814. 
11 Castelblanco E, Santacana M, Valls J et al. Usefulness of negative and weak-diffuse pattern of SDHB immunostaining in assessment of SDH mutations in paragangliomas and pheochromocytomas. Endocr Pathol 2013;24:199-205.

12 Martins RG, Nunes JB, Máximo V et al. A founder SDHB mutation in Portuguese paraganglioma patients. Endocr Relat Cancer 2013;20:L23-L26.

13 Sjursen W, Halvorsen H, Hofsli E et al. Mutation screening in a Norwegian cohort with pheochromocytoma. Fam Cancer 2013;12:529-535.

14 Lefebvre S, Borson-Chazot F, Boutry-Kryza $\mathrm{N}$ et al. Screening of mutations in genes that predispose to hereditary paragangliomas and pheochromocytomas. Horm Metab Res 2012;44:334-338.

15 Wang CP, Chen TC, Chang YL et al. Common genetic mutations in the start codon of the SDH subunit D gene among Chinese families with familial head and neck paragangliomas. Oral Oncol 2012;48:125-129.

16 Millar AC, Mete O, Cusimano RJ et al. Functional cardiac paraganglioma associated with a rare SDHC mutation. Endocr Pathol 2014;25:315-320.

17 Korpershoek E, Favier J, Gaal J et al. SDHA immunohistochemistry detects germline SDHA gene mutations in apparently sporadic paragangliomas and pheochromocytomas. I Clin Endocrinol Metab 2011;96:E1472-E1476.

18 Burnichon N, Brière JJ, Libé R et al. SDHA is a tumor suppressor gene causing paraganglioma. Hum Mol Genet 2010;19:3011-3020.

19 Welander J, Garvin S, Bohnmark R et al. Germline SDHA mutation detected by next-generation sequencing in a young index patient with large paraganglioma. J Clin Endocrinol Metab 2013;98:E1379-E1380.

20 Mason EF, Sadow PM, Wagner AJ et al. Identification of succinate dehydrogenase-deficient bladder paragangliomas. Am J Surg Pathol 2013;37:1612-1618.

21 Wagner AJ, Remillard SP, Zhang YX et al. Loss of expression of SDHA predicts SDHA mutations in gastrointestinal stromal tumors. Mod Pathol 2013;26:289-294.

22 Miettinen M, Killian JK, Wang ZF et al. Immunohistochemical loss of succinate dehydrogenase subunit A (SDHA) in gastrointestinal stromal tumors (GISTs) signals SDHA germline mutation. Am J Surg Pathol 2013;37:234-240.

23 Dwight T, Benn DE, Clarkson A et al. Loss of SDHA expression identifies SDHA mutations in succinate dehydrogenase-deficient gastrointestinal stromal tumors. Am J Surg Pathol 2013;37:226-233.

24 Oudijk L, Gaal J, Korpershoek E et al. SDHA mutations in adult and pediatric wild-type gastrointestinal stromal tumors. Mod Pathol 2013;26:456-463.

25 Italiano A, Chen CL, Sung YS et al. SDHA loss of function mutations in a subset of young adult wild-type gastrointestinal stromal tumors. BMC Cancer 2012;12:408.

26 Pantaleo MA, Astolfi A, Urbini M et al. Analysis of all subunits, SDHA, SDHB, SDHC, SDHD, of the succinate dehydrogenase complex in KIT/PDGFRA wildtype GIST. Eur J Hum Genet 2014;22:32-39.

27 Belinsky MG, Rink L, Flieder DB et al. Overexpression of insulin-like growth factor 1 receptor and frequent mutational inactivation of SDHA in wild-type SDHBnegative gastrointestinal stromal tumors. Genes Chromosomes Cancer 2013;52:214-224.

28 Janeway KA, Kim SY, Lodish M et al. Defects in succinate dehydrogenase in gastrointestinal stromal tumors lacking KIT and PDGFRA mutations. Proc Natl Acad Sci USA 2011;108:314-318.
29 Gaal J, Stratakis CA, Carney JA et al. SDHB immunohistochemistry: a useful tool in the diagnosis of CarneyStratakis and Carney triad gastrointestinal stromal tumors. Mod Pathol 2011;24:147-151.

30 Celestino R, Lima J, Faustino A et al. A novel germline SDHB mutation in a gastrointestinal stromal tumor patient without bona fide features of the CarneyStratakis dyad. Fam Cancer 2012;11:189-194.

31 Tenorio Jiménez C, Izatt L, Chang $\mathrm{F}$ et al. Carney Stratakis syndrome in a patient with SDHD mutation. Endocr Pathol 2012;23:181-186.

32 Gill AJ, Lipton L, Taylor J et al. Germline SDHC mutation presenting as recurrent SDH deficient GIST and renal carcinoma. Pathology 2013;45:689-691.

33 Gill AJ, Pachter NS, Chou A et al. Renal tumors associated with germline SDHB mutation show distinctive morphology. Am J Surg Pathol 2011;35:1578-1585.

34 Gill AJ, Pachter NS, Clarkson A et al. Renal tumors and hereditary pheochromocytoma-paraganglioma syndrome type 4. N Engl J Med 2011;364:885-886.

35 Paik JY, Toon CW, Benn DE et al. Renal carcinoma associated with succinate dehydrogenase B mutation: a new and unique subtype of renal carcinoma. J Clin Oncol 2014;32:e10-e13.

36 Malinoc A, Sullivan M, Wiech $\mathrm{T}$ et al. Biallelic inactivation of the SDHC gene in renal carcinoma associated with paraganglioma syndrome type 3 . Endocr Relat Cancer 2012;19:283-290.

37 Xekouki P, Pacak K, Almeida M et al. Succinate dehydrogenase (SDH) D subunit (SDHD) inactivation in a growth-hormone-producing pituitary tumor: a new association for SDH? J Clin Endocrinol Metab 2012;97: E357-E366.

38 Dwight T, Mann K, Benn DE et al. Familial SDHA mutation associated with pituitary adenoma and pheochromocytoma/paraganglioma. J Clin Endocrinol Metab 2013;98:E1103-E1108.

39 Gill AJ, Toon CW, Clarkson A et al. Succinate dehydrogenase deficiency is rare in pituitary adenomas. Am J Surg Pathol 2014;38:560-566.

40 Gill AJ, Chou A, Vilain R et al. Immunohistochemistry for SDHB divides gastrointestinal stromal tumors (GISTs) into two distinct types. Am J Surg Pathol 2010;34:636-644.

41 Williamson SR, Eble JN, Amin MB et al. Succinate dehydrogenase-deficient renal cell carcinoma: detailed characterization of 11 tumors defining a unique subtype of renal cell carcinoma. Mod Pathol 2014;28: 80-94.

42 Haller F, Moskalev EA, Faucz FR et al. Aberrant DNA hypermethylation of SDHC: a novel mechanism of tumor development in Carney triad. Endocr Relat Cancer 2014;21:567-577.

43 Rattenberry E, Vialard L, Yeung A et al. A comprehensive next generation sequencing-based genetic testing strategy to improve diagnosis of inherited pheochromocytoma and paraganglioma. J Clin Endocrinol Metab 2013;98:E1248-E1256.

44 McInerney-Leo AM, Marshall MS, Gardiner B et al. Whole exome sequencing is an efficient and sensitive method for detection of germline mutations in patients with phaeochromcytomas and paragangliomas. Clin Endocrinol (Oxf) 2014;80:25-33.

45 Crona J, Verdugo AD, Granberg D et al. Next-generation sequencing in the clinical genetic screening of patients with pheochromocytoma and paraganglioma. Endocr Connect 2013;2:104-111. 
46 Phaeochromocytoma Study Group in Japan, Kimura N, Takayanagi R, Takizawa N et al. Pathologic grading for predicting metastasis in phaeochromocytoma and paraganglioma. Endocr Relat Cancer 2014;21:405-414.

47 Landis JR, Koch GG. The measurement of observer agreement for categorical data. Biometrics 1977;33: 159-174.

48 Chan JK, Ip YT, Cheuk W. The utility of immunohistochemistry for providing genetic information on tumors. Int J Surg Pathol 2013;21:455-475.

49 Barletta JA, Bellizzi AM, Hornick JL. Immunohistochemical staining of thyroidectomy specimens for PTEN can aid in the identification of patients with Cowden syndrome. Am J Surg Pathol 2011;35:1505-1511.

50 Gaujoux S, Grabar S, Fassnacht M et al. $\beta$-catenin activation is associated with specific clinical and pathologic characteristics and a poor outcome in adrenocortical carcinoma. Clin Cancer Res 2011;17:328-336.

51 Ragazzon B, Libé R, Gaujoux S et al. Transcriptome analysis reveals that p53 and \{beta\}-catenin alterations occur in a group of aggressive adrenocortical cancers. Cancer Res 2010;70:8276-8281.

52 Waldmann J, Patsalis N, Fendrich V et al. Clinical impact of TP53 alterations in adrenocortical carcinomas. Langenbecks Arch Surg 2012;397:209-216.

53 Raymond VM, Everett JN, Furtado LV et al. Adrenocortical carcinoma is a lynch syndrome-associated cancer. J Clin Oncol 2013;31:3012-3018.

54 Gill AJ. Understanding the genetic basis of parathyroid carcinoma. Endocr Pathol 2014;25:30-34.

55 Kim MJ, Choi J, Khang SK et al. Primary intraosseous melanotic schwannoma of the fibula associated with the Carney complex. Pathol Int 2006;56:538-542.

56 Zembowicz A, Knoepp SM, Bei T et al. Loss of expression of protein kinase a regulatory subunit 1alpha in pigmented epithelioid melanocytoma but not in melanoma or other melanocytic lesions. Am J Surg Pathol 2007;31:1764-1775.

57 Gaujoux S, Tissier F, Ragazzon B et al. Pancreatic ductal and acinar cell neoplasms in Carney complex: a possible new association. J Clin Endocrinol Metab 2011;96:E1888-E1895.

58 Castro-Vega LJ, Buffet A, De Cubas AA et al. Germline mutations in $\mathrm{FH}$ confer predisposition to malignant pheochromocytomas and paragangliomas. Hum Mol Genet 2014;23:2440-2446.

59 Comino-Méndez I, Gracia-Aznárez FJ, Schiavi F et al. Exome sequencing identifies MAX mutations as a cause of hereditary pheochromocytoma. Nat Genet 2011;43:663-667.

60 Carney JA. Carney triad. Front Horm Res 2013;41: 92-110.

61 Blank A, Schmitt AM, Korpershoek E et al. SDHB loss predicts malignancy in pheochromocytomas/sympathethic paragangliomas, but not through hypoxia signalling. Endocr Relat Cancer 2010;17:919-928.

62 Menara M, Oudijk L, Badoual C et al. SDHD immunohistochemistry: a new tool to validate SDHx mutations in pheochromocytoma/paraganglioma. J Clin Endocrinol Metab 2015;100:E287-E291.

63 Witkowski L, Carrot-Zhang J, Albrecht S et al. Germline and somatic SMARCA4 mutations characterize small cell carcinoma of the ovary, hypercalcemic type. Nat Genet 2014;46:438-443.

64 Mete O, Tischler AS, de Krijger R et al. Protocol for the examination of specimens from patients with pheochromocytomas and extra-adrenal paragangliomas. Arch Pathol Lab Med 2014;138:182-188.

65 Dahia PL, Ross KN, Wright ME et al. A HIF1alpha regulatory loop links hypoxia and mitochondrial signals in pheochromocytomas. PLoS Genet 2005;1:72-80.

66 Klarskov L, Ladelund S, Holck S et al. Interobserver variability in the evaluation of mismatch repair protein immunostaining. Hum Pathol 2010;41:1387-1396.

67 Hicks DG, Boyce BF. The challenge and importance of standardizing pre-analytical variables in surgical pathologyspecimens for clinical care and translational research. Biotech Histochem 2012;87:14-17.

68 Dowsett M, Nielsen TO, A'Hern R et al. Assessment of Ki67 in breast cancer: recommendations from the International Ki67 in Breast Cancer working group. J Natl Cancer Inst 2011;103:1656-1664.

69 Watson N, Grieu F, Morris M et al. Heterogeneous staining for mismatch repair proteins during population-based prescreening for hereditary nonpolyposis colorectal cancer. J Mol Diagn 2007;9:472-478.

70 Garg K, Broaddus RR, Soslow RA et al. Pathologic scoring of PTEN immunohistochemistry in endometrial carcinoma is highly reproducible. Int J Gynecol Pathol 2012;31:48-56.

71 King KS, Pacak K. Familial pheochromocytomas and paragangliomas. Mol Cell Endocrinol 2014;386:92-100.

72 Welander J, Söderkvist P, Gimm O. Genetics and clinical characteristics of hereditary pheochromocytomas and paragangliomas. Endocr Relat Cancer 2011;18: R253-R276.

73 Jafri M, Maher ER. The genetics of phaeochromocytoma: using clinical features to guide genetic testing. Eur J Endocrinol 2012;166:151-158.

74 Favier J, Amar L, Gimenez-Roqueplo AP. Paraganglioma and phaeochromocytoma: from genetics to personalized medicine. Nat Rev Endocrinol 2014;11: 101-111.

75 Kircher M, Witten DM, Jain P et al. A general framework for estimating the relative pathogenicity of human genetic variants. Nat Genet 2014;46:310-315.

76 Evenepoel L, Papathomas TG, Krol A et al. Toward an improved definition of the genetic and tumor spectrum associated with SDH germ-line mutations. Genet Med 2014; doi: 10.1038/gim.2014.162.

77 Canu L, Rapizzi E, Zampetti B et al. Pitfalls in genetic analysis of pheochromocytomas/paragangliomascase report. J Clin Endocrinol Metab 2014;99: 2321-2326.

78 Lendvai N, Pawlosky R, Bullova P et al. Succinate-tofumarate ratio as a new metabolic marker to detect the presence of SDHB/D-related paraganglioma: initial experimental and ex vivo findings. Endocrinology 2014;155:27-32.

79 Richter S, Peitzsch M, Rapizzi E et al. Krebs cycle metabolite profiling for identification and stratification of pheochromocytomas/paragangliomas due to succinate dehydrogenase deficiency. J Clin Endocrinol Metab 2014;99:3903-3911.

Supplementary Information accompanies the paper on Modern Pathology website (http://www.nature.com/ modpathol) 